\title{
Ligand-induced formation of transient dimers of mammalian 12/15-lipoxygenase: A key to allosteric behavior of this class of enzymes?
}

\author{
Igor Ivanov, ${ }^{1 \star}$ Weifeng Shang, ${ }^{2}$ Lea Toledo, ${ }^{3,4}$ Laura Masgrau, ${ }^{4}$ Dmitri I. Svergun, ${ }^{2}$ \\ Sabine Stehling, ${ }^{1}$ Hansel Gómez, ${ }^{4}$ Almerinda Di Venere, ${ }^{5}$ Giampiero Mei, ${ }^{5}$ José M. Lluch, ${ }^{3,4}$ \\ Ewa Skrzypczak-Jankun, ${ }^{6}$ Àngels González-Lafont, ${ }^{3,4}$ and Hartmut Kühn ${ }^{1 \star}$ \\ ${ }^{1}$ Institute of Biochemistry, Charité-Universitätsmedizin Berlin, D-13346 Berlin, Germany \\ 2 European Molecular Biology Laboratory Outstation, c/o DESY, D-22603 Hamburg, Germany \\ ${ }^{3}$ Departament de Química, Universitat Autònoma de Barcelona, 08193 Bellaterra, Spain \\ ${ }^{4}$ Institut de Biotecnologia i de Biomedicina (IBB), Universitat Autònoma de Barcelona, 08193 Bellaterra, Spain \\ ${ }^{5}$ IRCCS Neuromed, Pozzilli, and Department of Experimental Medicine and Biochemical Sciences, University of Tor Vergata, \\ 00133 Rome, Italy \\ ${ }^{6}$ Urology Research Center, College of Medicine, University of Toledo, Toledo, Ohio 43614
}

\begin{abstract}
Mammalian lipoxygenases (LOXs) have been implicated in cellular defense response and are important for physiological homeostasis. Since their discovery, LOXs have been believed to function as monomeric enzymes that exhibit allosteric properties. In aqueous solutions, the rabbit 12/15LOX is mainly present as hydrated monomer but changes in the local physiochemical environment suggested a monomer-dimer equilibrium. Because the allosteric character of the enzyme can hardly be explained using a single ligand binding-site model, we proposed that the binding of allosteric effectors may shift the monomer-dimer equilibrium toward dimer formation. To test this hypothesis, we explored the impact of an allosteric effector [13(S)hydroxyoctadeca-9 $(Z), 11(E)$-dienoic acid] on the structural properties of rabbit $12 / 15$-LOX by small-angle X-ray scattering. Our data indicate that the enzyme undergoes ligand-induced dimerization in aqueous solution, and molecular dynamics simulations suggested that LOX dimers may be stable in the presence of substrate fatty acids. These data provide direct structural evidence for the existence of LOX dimers, where two noncovalently linked enzyme molecules might work in unison and, therefore, such mode of association might be related to the allosteric character of 12/15-LOX. Introduction of negatively charged residues (W181E + H585E and $\mathrm{L} 183 \mathrm{E}+\mathrm{L} 192 \mathrm{E})$ at the intermonomer interface disturbs the hydrophobic dimer interaction of the wild-type LOX, and this structural alteration may lead to functional distortion of mutant enzymes.
\end{abstract}

Proteins 2012; 80:703-712.

(C) 2011 Wiley Periodicals, Inc.

Key words: lipoxygenases; allosterism; transient proteinprotein interaction; $\mathrm{X}$-ray small-angle scattering; molecular dynamics.

\section{INTRODUCTION}

Lipoxygenases (LOXs) constitute a family of lipid peroxidizing enzymes, which metabolize polyunsaturated acids to biologically active mediators. Mammalian LOXs and their products have been implicated in cellular defense response and in numerous pathophysiological conditions. ${ }^{1-4}$ Since their discovery, 12/15-LOXs have been believed to function as monomers possessing a single binding site for substrate and product. Experiments with 15(S)-hydroperoxyeicosa$5(Z), 8(Z), 11(Z), 13(E)$-tetraenoic acid (15(S)-HpETE) indeed indicated covalent linkage of this endogenous LOX product to rabbit 12/15-LOX active-site peptides with a stoichiometry close to equimolar. ${ }^{5}$ Earlier investigations into the solution structure of 12/15-LOX have shown that the rabbit enzyme occurs mainly as hydrated monomer 6 supporting the traditional concept of LOX catalysis. ${ }^{7}$ However, more recent small-angle X-ray scattering (SAXS) experiments suggested the possibility of a monomer-dimer equilibrium between $12 / 15$-LOX protomers, ${ }^{8}$ which is controlled by the physiochemical environment (ionic strength)

Additional Supporting Information may be found in the online version of this article.

Abbreviations: 13(S)-HODE, 13(S)-hydroxyoctadeca-9(Z),11(E)-dienoic acid; 15(S)HpETE, 15(S)-hydroperoxyeicosa-5(Z),8(Z),11(Z),13(E)-tetraenoic acid; 13(S)-HpODE, $13(S)$-hydroperoxyoctadeca- $9(Z), 11(E)$-dienoic acid; LA, linoleic acid; LOX(s), lipoxyge-

Grant sponsor: European Molecular Biology Laboratory, Hamburg Outstation; Grant numbers: SAXS-09-036, SAXS-10-004; Grant sponsor: Spanish "Ministerio de Ciencia e Innovación"; Grant number: CTQ2008-02403/BQU; Grant sponsor: Generalitat de Catalunya; Grant number: 2009SGR409; Grant sponsor: DFG; Grant number: GRK1673/1; Grant sponsor: "Ramon y Cajal" Program

${ }^{\star}$ Correspondence to: Igor Ivanov or Hartmut Kuhn, Institute of Biochemistry, CharitéUniversitätsmedizin Berlin, Oudenarder Str. 16, D-13346 Berlin, Germany.

E-mail: igor_ivanov@gmx.de or hartmut.kuehn@charite.de

Received 20 June 2011; Revised 3 October 2011; Accepted 10 October 2011 Published online 19 October 2011 in Wiley Online Library (wileyonlinelibrary.com). DOI: $10.1002 /$ prot. 23227 
and the protein concentration. In contrast to the observations made in solution, the crystallographic 12/15LOX-inhibitor complex ${ }^{9}$ represents a dimer, which consists of two structurally different conformers A and B that are associated via their $\alpha 2$ helixes. In this structure (PDB 2P0M entry), one monomer (conformer B) carries the inhibitor within the putative substrate-binding pocket, whereas the other one is not liganded (conformer A). It is still unclear whether the intermolecular contacts in the crystals represent a biologically relevant structure or may be considered as an "in vitro artifact" induced during crystal grown at high ionic strength. If dimerization is biologically relevant, active-site ligands might play a role as molecular trigger that augments the weak protein-protein interaction between 12/15-LOX molecules forcing dimer formation. Modeling of the interface contacts between two 12/15-LOX molecules suggested that the geometry reported in the crystals for dimers (A:B) appears to be thermodynamically the most favorable with a solvation free energy of $-25 \mathrm{kcal} / \mathrm{mol}^{8}{ }^{8}$

There are numerous examples in the literature characterizing the cross-talk between monomeric subunits of highly organized protein oligomers. Such protein-protein interactions frequently involve both weak and stronger transient associates, in which obligate homodimers as well as nonobligate heterodimer complexes are present. ${ }^{10-13}$ An excellent example of such cooperativity between the two subunits of obligate homodimeric protein has recently been ascribed to cyclooxygenase-2 (COX-2), another lipid peroxidizing enzyme. In COX-2, one monomer acts as an "allosteric" subunit modulating the catalytic efficiency of its partner monomer. ${ }^{14,15}$ Also, mammalian LOXs have been shown to exhibit allosteric properties. Although functional data suggest that binding of endogenous product, 13(S)-H(p)ODE, appears to lock the enzyme in a catalytically competent state, the $13(S)$ $\mathrm{H}(\mathrm{p})$ ODE-binding region(s) have not been identified. ${ }^{16,17}$ Because the allosteric character of the enzyme can hardly be explained using a single ligand binding-site model, we proposed that the binding of allosteric effectors such as $13(S)-\mathrm{H}(\mathrm{p}) \mathrm{ODE}$ may induce conformational changes so that transient dimeric complex may be formed.

To explore whether binding of allosteric effectors may induce intermolecular association of 12/15-LOX protomers in aqueous solutions, we applied SAXS. To diminish unwanted side effects and unspecific ligand binding to the protein, we refrained from using 13(S)-hydroperoxyoctadeca-9 $(Z), 11(E)$-dienoic acid $(13(S)$-HpODE) as allosteric effector but selected 13(S)-hydroxyoctadeca$9(Z), 11(E)$-dienoic acid (13(S)-HODE) instead. Unlike the situation with the enzymatic product $13(S)$-HpODE, examining of the cross-talk between the 12/15-LOX protomers in the presence of fatty acid substrate is technically more challenging owing to the high interconversion rate of these natural ligands. Previous docking calcula- tions combined with molecular dynamics (MD) simulations on the rabbit 12/15-LOX-arachidonic acid (AA) complex suggested that enzyme-substrate interaction induces similar structural alterations as observed in the crystallographic conformer B upon inhibitor binding. ${ }^{18}$ To find out whether 12/15-LOX is able to form transient intermolecular associates that are also stable in the presence of substrate, we created in silico dimer models (where conformer A is an "empty" molecule and conformer B contains $\mathrm{AA}$ at the active site) and performed MD simulations.

\section{MATERIALS AND METHODS}

\section{Protein preparation}

The recombinant wild-type rabbit $12 / 15$-LOX and the mutants were expressed in E. coli as His-tagged fusion and purified using affinity chromatography on nickelagarose followed by anion-exchange and size exclusion chromatography columns as described in protocol. ${ }^{8}$ After gel filtration, the corresponding protein solutions in 20 $\mathrm{mM}$ Tris- $\mathrm{HCl}$ buffer, $\mathrm{pH} 8.0$ were concentrated either to 0.7 or to $1.0 \mathrm{mg} / \mathrm{mL}$ with a Spin- $\mathrm{X}^{\mathrm{R}} 630 \mathrm{k}$ MWCO concentrator (Corning, UK) and frozen in the presence of $5 \%$ (vol.) glycerol in liquid nitrogen. The protein concentration was estimated spectrophotometrically at $280 \mathrm{~nm}$ using molecular extinction coefficients $1.78 \mathrm{mg} /(\mathrm{mL} \mathrm{cm})$ for the wild-type enzyme and the L183E + L192E mutant but $1.70 \mathrm{mg} /(\mathrm{mL} \mathrm{cm})$ for the W181E + H585E mutant. Enzyme-ligand complexes were prepared immediately before each SAXS measurement by adding the corresponding amounts of a $47 \mathrm{mM} 13(S)$-HODE solution to the protein sample. The mixture was incubated for 15 min on ice and centrifuged at $14,000 \mathrm{~g}$ for $30 \mathrm{~min}$ at $5^{\circ} \mathrm{C}$.

\section{SAXS data collection and analysis}

The SAXS measurements were performed at the EMBL BioSAXS beamline X33 at the DORIS storage ring 19 (DESY, Hamburg) using the X-ray wavelength of $1.54 \AA$ and the sample-to-detector distance of $2.7 \mathrm{~m}$. A noisefree photon-counting PILATUS detector was used to record four consecutive frames of $30 \mathrm{~s}$, which were averaged after confirming the absence of radiation damage. An automatic sample changer was used in all measurements to facilitate loading the samples and cleaning the cell windows made of mica or polycarbonate films. 20 The scattering patterns of the corresponding buffer solutions were recorded before and after the measurements of the protein sample, and then the averaged buffer patterns were subtracted from the protein patterns. Bovine serum albumin solution of $4.6 \mathrm{mg} / \mathrm{mL}$ in $50 \mathrm{mM}$ Hepes, $\mathrm{pH} 7.5$, was used for calibration of the molecular mass. The samples of wild-type rabbit 12/15-LOX, its mutants, and the enzyme-ligand complexes were kept at $10^{\circ} \mathrm{C}$ and 
measured at the protein concentration of $0.7 \mathrm{mg} / \mathrm{mL}$ for the wild-type enzyme, $0.7 \mathrm{mg} / \mathrm{mL}$ for the W181E + H585E mutant, and $1.0 \mathrm{mg} / \mathrm{mL}$ for the L183E + L192E mutant. Several independently prepared solutes of protein samples were applied for the measurements, and only those that were not affected by aggregation were considered for further analysis. All samples for the rabbit LOX were scattered to a nominal resolution of $30 \AA$. Data reduction and Guinier analysis were performed using the program PRIMUS. ${ }^{21}$ For polydisperse samples, an improved version of the SASREF program was used, ${ }^{22}$ allowing the simultaneous consideration of polydispersity and free movement of defined rigid subunits. The radius of gyration for the wild-type enzyme in the crystal lattice was evaluated from the crystal structure $2 \mathrm{P} 0 \mathrm{M}$ using the program CRYSOL. ${ }^{23}$ To assess the volume fractions of the individual species in the mixture, the SAXS curve was represented by a linear combination of the theoretical scattering profiles calculated from the crystal structures or the rigid body models of the relevant components in solution using the program OLIGOMER. ${ }^{21}$ The structure of the conformer A was refined using two major domains (A:2-108|A:109-663), and the dimer (A:2-108|A:109-663, A109-663|A:2-108) was generated via P2 symmetry operation. The catalytic domains (109-663) were fixed to keep the dimer interface similar to that in the crystal $(2 \mathrm{P} 0 \mathrm{M})$, but the N-terminal domains (2-108) were allowed to move freely. Additionally, a tetramer model in P222 symmetry for the ligand-bound L183E + L192E mutant was constructed using the program SASREF, ${ }^{22}$ and the volume fractions for the ligand free mutant were assessed using the program OLIGOMER. ${ }^{21}$

\section{Kinetic assay system}

Oxygenation kinetics were assayed by absorption spectrometry (Shimadzu 2100 spectrophotometer) measuring the increase of absorbance at $235 \mathrm{~nm}$ in the substrate concentration range between 1 and $50 \mu M$. The assay mixture was a phosphate buffered saline (PBS), $\mathrm{pH}$ 7.4, containing either $56 \mathrm{nM}$ or $165 \mathrm{n} M$ enzyme, normalized to a 1:1 stoichiometry of apoenzyme and nonheme iron. The reaction was started by addition of substrate. All measurements were carried out at room temperature.

\section{Molecular dynamics simulations of the enzyme-substrate complex for wild-type and double mutant dimers}

Homodimeric models (conformer A and B) of 12/15LOX with AA bound in the conformer B were built for the wild-type enzyme and the W181E $+\mathrm{H} 585 \mathrm{E}$ and L183E + L192E double mutants. The initial coordinates for the wild-type enzyme dimer were taken from the crystallographic structure (PDB entry code 2P0M). Crystallographic water molecules were also considered in the model. The $\mathrm{Fe}(\mathrm{III})-\mathrm{OH}^{-}$cofactor was built in both monomers considering the octahedral coordination of the iron. AA was positioned inside the active site according to the docking results obtained in our previous work on conformer B. ${ }^{18}$ The missing hydrogen atoms were built with the HBUILD facility in the CHARMM package (version c35). ${ }^{24}$ The construction of the two double mutants (W181E + H585E and L183E + L192E) was done in silico by changing the side chain of the mutated residues in the wild-type model and searching for the best rotamer in each case. The program Chimera-version1.5.2 25 was used for this purpose. Each of the dimeric models was submitted to MD simulations to get an indicator of the stability of the interface and compare mutated enzymes with the wild-type one. The complexes were solvated with a $151 \AA \times 121 \AA \times 73 \AA$ box of preequilibrated TIP 3 waters. The total charge of the systems was neutralized with the addition of sodium cations. The resulting solvated dimeric systems had a total of $\sim 141,713$ atoms, among them $\sim 41,497$ atoms belong to the 1326-amino acid dimer. The simulations were performed at constant pressure and temperature using periodic boundary conditions. The extended system constant pressure as well as the Hoover constant temperature algorithms ${ }^{26}$ were used. The PBC was built with the CRYSTAL module of CHARMM using an orthorhombic unit cell, and the Particle-Mesh Ewald method was used for long-range electrostatics. A time step of $2 \mathrm{fs}$ was used with all the angles and bonds involving hydrogen atoms constrained by the SHAKE algorithm. ${ }^{27}$ All the systems were first submitted to some energy minimization steps to relax them. They were then heated and equilibrated to reach the desired temperature $(300 \mathrm{~K})$. Restrains were applied to the protein and the ligand, which were gradually released to prevent inaccurate behaviors. Finally, a production period of 4 ns was run. Two independent 4ns simulations, which differ in the initial assignment of velocities, were run for each dimer. The simulations were done using the CHARMM-22 force field parameters 28,29 for the protein atoms, for the iron and its first coordination sphere we used the parameters specifically developed in a previous work ${ }^{30}$ for the same system. The force field topology and parameters for the lipid substrate were derived from the lipids CHARMM-27 ones. ${ }^{31}$

\section{RESULTS AND DISCUSSION}

\section{Wild-type 12/15-LOX solution structure in the presence and absence of an allosteric effector}

Before SAXS measurements, we tested the impact of different 13(S)-HODE concentrations on particle size distribution of wild-type 12/15-LOX in the range between 10 and $100 \mu M$ using dynamic light scattering. For the wild-type enzyme $(1 \mu M)$, a notable increase in the dia- 

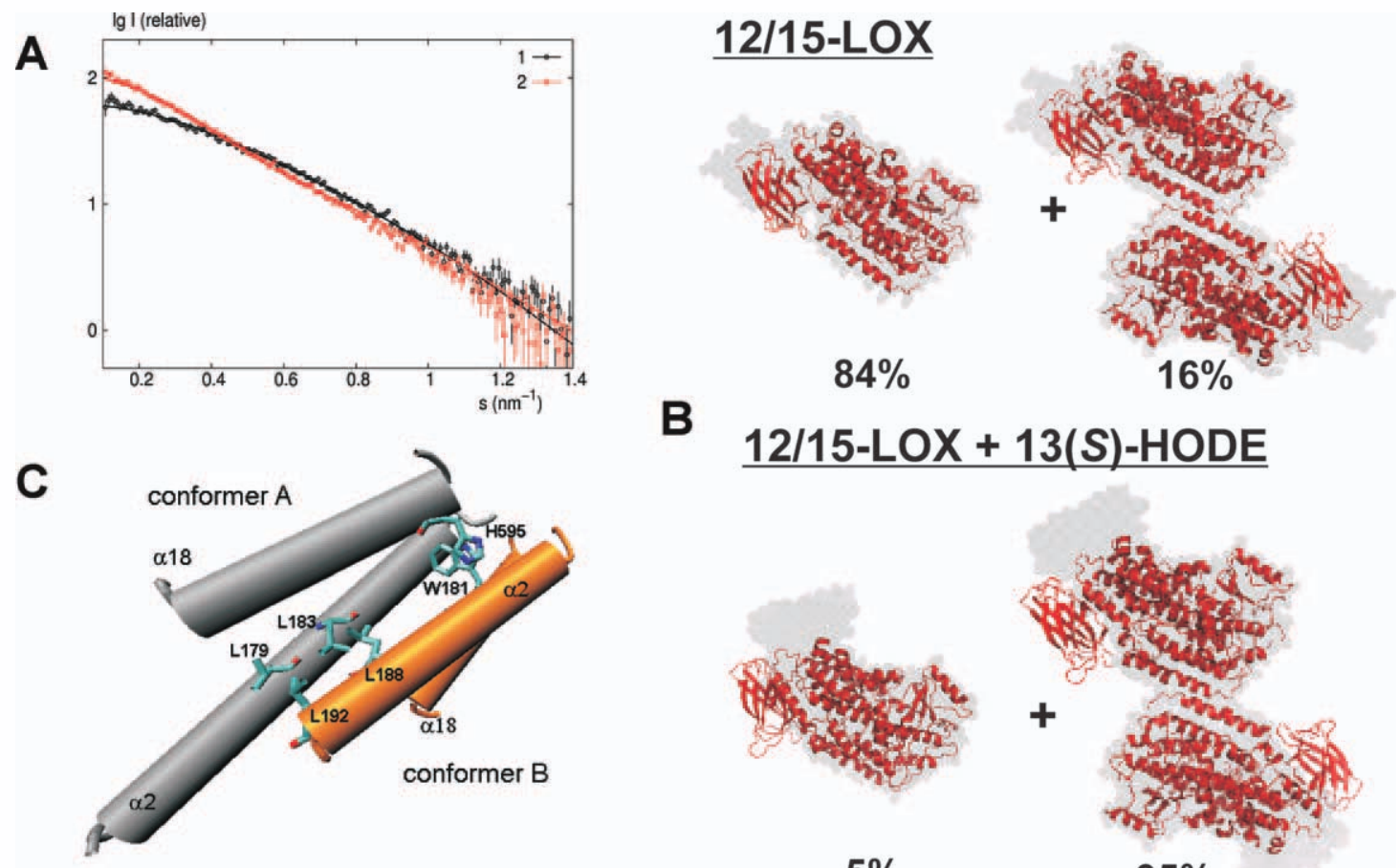

B
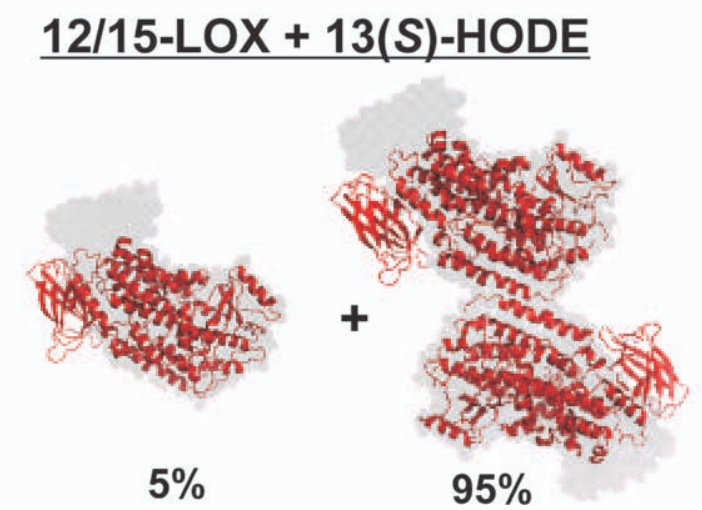

\section{Figure 1}

Experimental SAXS data and the dimer interface. (A) Experimental SAXS patterns and fits by linear combinations of the scattering from the refined monomer and dimer for the ligand-free wild-type 12/15-LOX (black circles and line 1) and 12/15-LOX with ligand (red squares and line 2). (B) Comparison of the crystal structures of the monomer and dimer (red ribbon) as in 2P0M and the models obtained from rigid body refinement (gray spheres) for the wild-type rabbit 12/15-LOX without ligand (upper panel) and in the presence of 10-fold molar excess of 13 (S)-HODE as ligand (lower panel). The dimers are generated by applying the P2 symmetry on the conformers A while keeping the dimeric interface similar to that in the crystal structure (PDB code: $2 \mathrm{P0M}$ ). (C) The residues contributing to interaction between ligand-free (conformer A) and ligand-bound (conformer B) molecules in 12/15-LOX dimer. The program VMD ${ }^{35} 1.4 .8$ version (University of Illinois) and the coordinates of rabbit LOX complex (PDB code: $2 \mathrm{P} 0 \mathrm{M}$ ) were used to create this figure.

meter of particles was observed in the range between 10 and $40 \mu M$ of $13(S)$-HODE (Supporting Information Fig. S1). SAXS measurements were performed first for different wild-type 12/15-LOX preparations in the absence of 13(S)-HODE, and only those samples not affected by protein aggregation were selected for further experimentation. Then, 13(S)-HODE was added at different concentrations and SAXS measurements were repeated. Evaluation of the scattering patterns of ligand-free 12/15LOX yielded a radius of gyration of $R_{\mathrm{g}}=3.1 \pm 0.1 \mathrm{~nm}$, which is higher than the theoretical value of $2.86 \mathrm{~nm}$ calculated for the crystallographic conformer A (2P0M). When the protein was incubated with a 10-fold molar excess of $13(S)$-HODE, $R_{\mathrm{g}}$ increased to $4.2 \pm 0.1 \mathrm{~nm}$, a value that is close to the theoretical $R_{\mathrm{g}}(4.11 \mathrm{~nm})$ calculated for the crystal dimer (conformers A:B). Comparison of experimental and theoretical scattering patterns estimated for the crystal monomer and dimer structures revealed noticeable discrepancies in the region of $s<0.3$ $\mathrm{nm}^{-1}$ for both ligand-free enzyme and ligand-containing samples. In addition, we observed systematic deviations in the $s$-range between 0.6 and $1 \mathrm{~nm}^{-1}$ in the sample with ligand (Supporting Information Fig. S2). Considering the absence of any signs of unspecific protein aggregation, this mismatch may be explained by a monomerdimer equilibrium and/or by the movement of the flexible N-terminal domain relative to catalytic subunit (interdomain movement). ${ }^{8}$ By using rigid body modeling $^{22}$ a linear combination of the calculated scattering curves for the conformer A and P2 symmetry-related dimer models were fitted to the experimental data [Fig. $1(\mathrm{~A})$ ], and the following conclusions were drawn: (i) The major volume fraction of ligand-free wild-type enzyme in solution was monomeric $(\sim 85 \%)$ and only $\sim 15 \%$ of the enzyme was present as dimer. (ii) In the presence of a 10-fold molar excess of 13(S)-HODE, the volume fraction of the dimer increased to $\sim 95 \%$. Thus, in the presence of the allosteric ligand, the monomer-dimer equilibrium was shifted almost completely toward enzyme dimers. (iii) In the refined monomer and dimer models, the N-terminal domain appears to swing away from catalytic unit resulting in a significant increase in the size of enzyme monomers and dimers [Fig. 1(B)]. This result, which is in accordance with our previous observations of 
Transient Dimers of Mammalian 12/15-Lipoxygenase

Table I

Analysis of the SAXS Data Obtained for the Wild-Type 12/15-LOX and Its W181E + H585E Mutant

\begin{tabular}{|c|c|c|c|c|c|c|c|c|}
\hline \multirow[b]{2}{*}{$\begin{array}{l}\text { Excess ligand } \\
\text { amount (mol) }\end{array}$} & \multicolumn{4}{|c|}{ Wild-type 12/15-LOX } & \multicolumn{4}{|c|}{ Mutant (W181E + H585E) } \\
\hline & $\chi^{\mathrm{a}}$ & $<\mathrm{R}_{g}{ }^{\mathrm{b}}>(\mathrm{nm})$ & $\begin{array}{c}\text { Monomer volume } \\
\text { fraction (\%) }\end{array}$ & $\begin{array}{c}\text { Dimer volume } \\
\text { fraction (\%) }\end{array}$ & $\chi$ & $<\mathrm{R}_{g}>(n m)$ & $\begin{array}{c}\text { Monomer } \\
\text { volume fraction (\%) }\end{array}$ & $\begin{array}{c}\text { Dimer volume } \\
\text { fraction }(\%)\end{array}$ \\
\hline 0 & 1.38 & $3.3 \pm 0.1$ & 84 & 16 & 1.15 & $3.2 \pm 0.1$ & 86 & 14 \\
\hline 40 & 1.09 & $4.5 \pm 0.1$ & 23 & 77 & 1.01 & $3.4 \pm 0.1$ & 77 & 23 \\
\hline
\end{tabular}

${ }^{\mathrm{a}}$ Discrepancy factor $\chi$.

${ }^{b}$ Average radius of gyration as well as volume fractions of the monomer/dimer equilibrium for the wild-type 12/15-LOX and the W181E + H585E mutant. The measurements were performed either with or without ligand. $R_{\mathrm{g}}$ values were estimated using the program OLIGOMER.

motional flexibility of the $\mathrm{N}$-terminal $\beta$-barrel, suggested that interdomain interface residues might be involved in substrate/ligand binding. ${ }^{32}$ (iv) At higher ligand concentrations (40-fold molar excess), the volume fraction of the enzyme dimer slightly decreased to about $80 \%$ (Table I). Although the mechanistic basis for this decrease has not been explored, it might be related to repulsion between two B-conformers, when more than 50\% of LOX molecules are present as liganded B-form. Although smaller structural elements involved in the interaction between two molecules remain undistinguishable at $30 \AA$, the low-resolution models obtained clearly indicate an overall molecular shape change for the 12/15-LOX:13(S)HODE complex when compared with ligand-free 12/15LOX.

\section{SAXS of the 12/15-LOX dimer interface mutants}

In rabbit 12/15-LOX crystallographic dimers L179, L183, L188, and L192 form a hydrophobic cluster, in which two leucine residues distanced by one $\alpha$-helix turn get in direct contact with the corresponding structural motif of the partner monomer that runs in opposite direction [Fig. 1(C)]. Such association resembles a leucine zipper motif, 33 a supersecondary structure that functions as dimerization domain generating adhesive forces between two monomers. In addition, W181 of the $\alpha 2$ helix, which has been implicated in membrane binding, 34 interacts with $\mathrm{H} 585$ of helix $\alpha 18$ [Fig. 1(C)] and, thus, may also contribute to stabilize the dimer interface. If these structural motives are involved in intermolecular association induced by 13(S)-HODE, introduction of charged residues at critical positions is expected to destabilize the entire hydrophobic bound network and thus may prevent dimerization. To find the most suitable candidates for in vitro site-directed mutagenesis studies, each of the residues that supposedly contributed to the hydrophobic interface (Supporting Information Fig. S3) was mutated in silico to the other 19 canonic proteinogenic amino acids. On the basis of the free energy calculations, we selected two amino acid combinations (L183E + L192E and W181E + H585E) for which monomer repulsion in silico was suggested (Supporting Information
Table SI), created the corresponding double mutants, and performed SAXS measurements to quantify the degree of LOX dimerization.

Addition of $13(S)$-HODE to the wild-type 12/15LOX induced enzyme dimerization as indicated by the alterations in the X-ray scattering pattern [Fig. 1(A)]. In contrast, when we compared the SAXS pattern of the $\mathrm{W} 181 \mathrm{E}+\mathrm{H} 585 \mathrm{E}$ mutant in the presence and absence of the allosteric ligand 13(S)-HODE [Fig. 2(A)], we did not observe significant differences. In fact, the calculated $R_{\mathrm{g}}$ values for this mutant measured either in the absence or presence of 13(S)-HODE were very similar to that obtained for the ligand-free wild-type enzyme (Table I).

For the ligand-free L183E + L192E double mutant a larger $R_{\mathrm{g}}=3.6 \pm 0.1 \mathrm{~nm}$ was obtained. When the protein was incubated with 13(S)-HODE, the experimental SAXS profile revealed significant changes in the entire $s$ range from 0.1 to $2.5 \mathrm{~nm}^{-1}$ [Fig. 2(B)]. Analysis of the SAXS data for the ligand-bound L183E + L192E mutant yields $R_{\mathrm{g}}=4.3 \pm 0.1 \mathrm{~nm}$ and $D_{\max }=13 \pm 1 \mathrm{~nm}$. Although these values are similar to those obtained from the crystal dimer (4.11 and $14 \mathrm{~nm}$, respectively), the distinct plateau in the $s$-range from 0.8 to $1.3 \mathrm{~nm}^{-1}$ of the SAXS curve suggests that the solute has a different shape [Fig. 2(B)]. The ab initio model reveals an excluded volume of $480 \pm 10 \mathrm{~nm}^{3}$, which is 2.5 times larger than that estimated for dimer from 2P0M $\left(190 \mathrm{~nm}^{3}\right)$. The molecular mass estimated from SAXS data constitutes $230 \pm 10 \mathrm{kDa}$, a value which is three times higher than the mass of $12 / 15$-LOX monomer $(75 \mathrm{kDa})$. These results suggest that enzyme oligomers (trimers or tetramers) appear to be present. Attempts to construct a stable trimer model were not successful, whereas a tetramer model containing the crystallographic conformer A as subunit and applying P222 symmetry did perfectly fit the experimental data with $\chi=1.08$ [Fig. 2(B)].

The $R_{\mathrm{g}}$ value calculated for the ligand-free L183E + L192E mutant was significantly larger than that obtained for the ligand-free wild-type 12/15-LOX. Assuming a steady state between monomers and dimers, this difference could be explained by an increase in the dimer fraction to $\sim 30 \%(\chi=1.16)$ (Table II). On the other hand, the experimental SAXS curve for the ligand-free L183E 

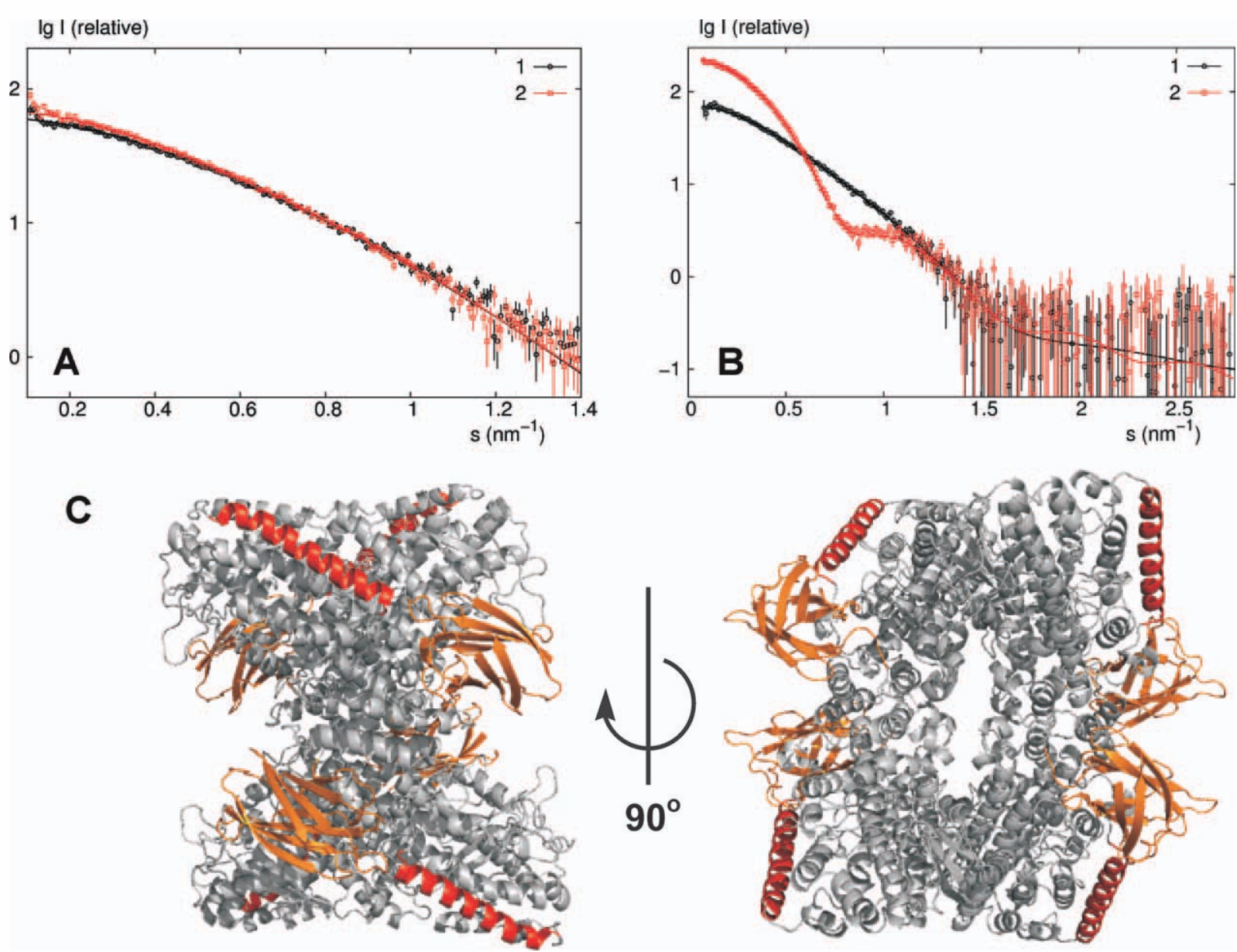

\section{Figure 2}

Experimental SAXS patterns and fits of models for the double mutants in a ligand-free form and as a complex with 13(S)-HODE. (A) SAXS patterns and the fitted models of refined monomers and dimers for the W181E + H585E mutant. The black (1) and the red (2) curves correspond to the samples before and after adding the 40-fold molar excess of 13(S)-HODE, respectively. (B) SAXS patterns and the fitted models of refined monomers and dimers for the L183E + L192E mutant. The curve 1 (black) corresponds to the SAXS pattern of ligand-free sample and fit by a linear combination of the scattering from the crystal monomer and a tetramer model in P222 symmetry. The curve 2 (red) corresponds to the sample with ligand and the fit of the tetramer model. (C) Tetramer model of L183E + L192E mutant complex with 13(S)-HODE in P222 symmetry. The four catalytic domains are shown in gray color, $\mathrm{N}$-terminal domains in orange, and $\alpha 2$ helixes in red. The structure shows significant difference to the dimer with P2 symmetry where both $\alpha 2$ helixes are tightly associated.

+ L192E mutant could be fitted with discrepancy factor of $\chi=1.23$ to a curve representing a mixture, in which $10 \%$ tetramers and $90 \%$ monomers were present. If one considers the prediction of monomer repulsion within a dimer as suggested by the free energy calculations (Supporting Information Table SI), the dimer model becomes

Table II

Analysis of the SAXS Data Obtained for the Wild-Type 12/15-LOX and Its L183E + L192E

\begin{tabular}{|c|c|c|c|c|c|c|}
\hline \multirow[b]{2}{*}{$\begin{array}{l}\text { Ligand amount } \\
\text { excess (mol) }\end{array}$} & \multicolumn{3}{|c|}{ Monomer-dimer equilibrium ${ }^{a}$} & \multicolumn{3}{|c|}{ Monomer-tetramer equilibrium ${ }^{b}$} \\
\hline & $\chi^{c}$ & $\begin{array}{l}\text { Monomer volume } \\
\text { fraction }(\%)\end{array}$ & $\begin{array}{l}\text { Dimer volume } \\
\text { fraction }(\%)\end{array}$ & $\chi$ & $\begin{array}{l}\text { Monomer volume } \\
\text { fraction }(\%)\end{array}$ & $\begin{array}{l}\text { Tetramer volume } \\
\text { fraction }(\%)\end{array}$ \\
\hline 0 & 1.16 & 71 & 29 & 1.23 & 91 & 9 \\
\hline 40 & - & - & - & 1.08 & 0 & 100 \\
\hline
\end{tabular}



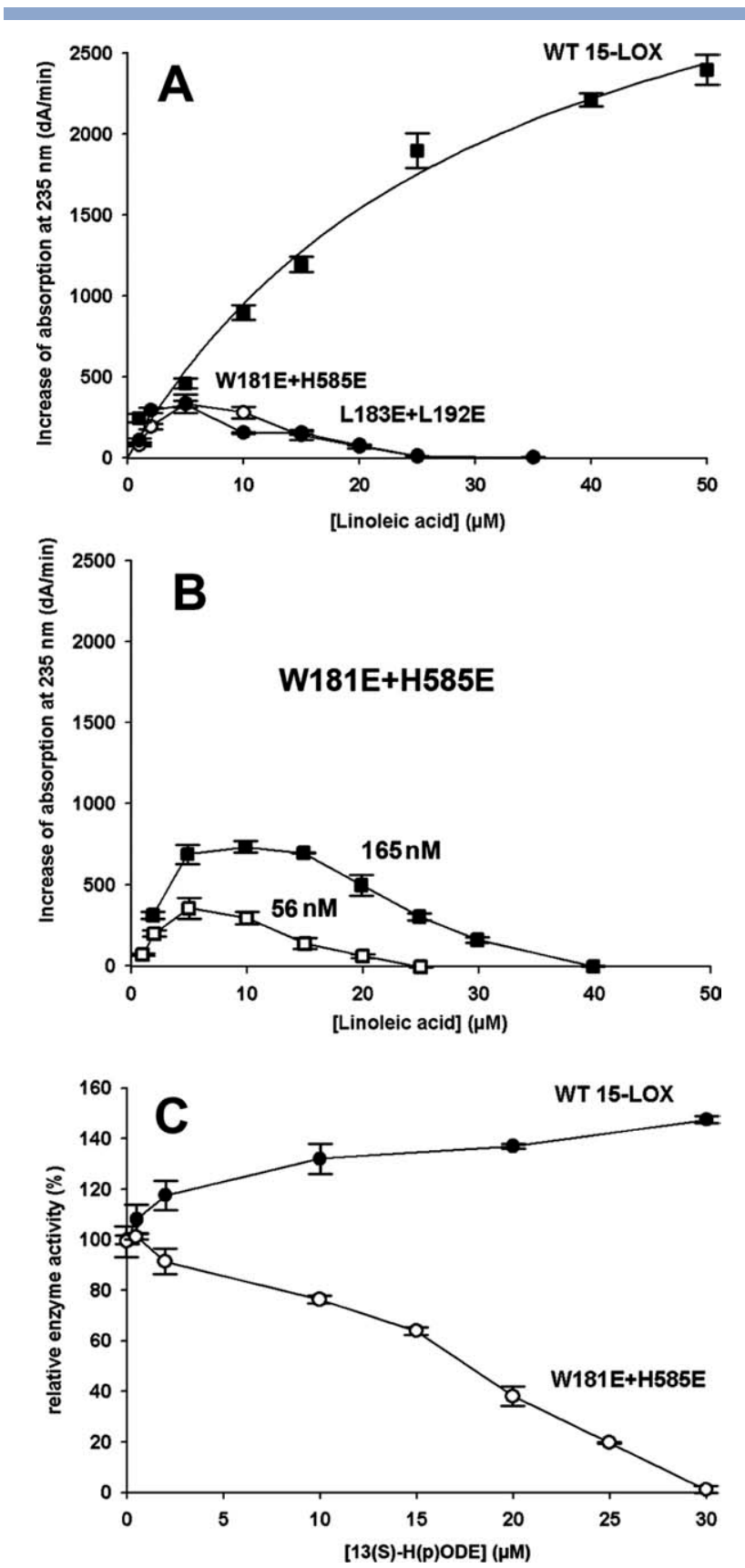

Figure 3

Oxygenation of linoleic acid by the recombinant rabbit 12/15-LOX and the dimer interface mutants. (A) The rate of conjugated diene formation measured at $235 \mathrm{~nm}$ is plotted versus linoleic acid concentration for the wild-type enzyme (black squares), W181E + H585E (open circles), and L183E + L192E (black circles) mutants. Enzyme concentration was normalized to iron content, and $56 \mathrm{nM}$ LOX solution in PBS was applied for each measurement. (B) The rate of conjugated diene formation at two different W181E + H585E mutant concentrations: $56 \mathrm{nM}$ (open squares) and $165 \mathrm{nM}$ (black squares). Linoleic acid was used as a substrate. (C) Relative catalytic activities of the wild-type 12/15-LOX and W181E + H585E mutant are plotted against concentration of allosteric activator $(13(S)-\mathrm{H}(\mathrm{p}) \mathrm{ODE})$. Wildtype enzyme (black circles) and W181E + H585E (open circles) 165 $\mathrm{n} M$ each were preincubated with different concentrations of 13(S)$\mathrm{H}(\mathrm{p}) \mathrm{ODE}$ for $30 \mathrm{~s}$ and the reaction was started with $10 \mu \mathrm{M}$ LA. Figures were prepared using Sigma Plot 11.0. less likely. Moreover, such model cannot be used to explain the experimental SAXS patterns obtained for L183E + L192E mutant in the presence of ligand. Structural analysis of the tetramer model suggests that the $\alpha 2$ helixes containing the mutated residues are not tightly associated [Fig. 2(C)]. Although this mutant forms larger oligomers in the presence of ligand and W181E + H585E does not, our SAXS data clearly indicate that mutations of the amino acids mentioned above may disrupt the original dimer interface present in the wild type.

\section{Kinetic assays reveal pronounced substrate/ product inactivation of the double mutants}

To explore the impact of disruption of the original dimer interface of rabbit 12/15-LOX on functional enzyme properties, we assayed the catalytic activity of the wild-type LOX and the double mutants (W181E + H585E and L183E + L192E) using linoleic acid (LA) as substrate. As shown in Figure 3(A), the wild-type enzyme follows Michaelis-Menten kinetics and a $K_{\mathrm{M}}$ of $21.3 \pm$ $3.6 \mu M$ and a maximum turnover rate at substrate saturation $\left(k_{\text {cat }}\right)$ of $47.23 \pm 2.83 \mathrm{~s}^{-1}$ were determined. In contrast, neither of the double mutants followed Michaelis-Menten kinetics. Instead, they were gradually inactivated at increasing substrate concentration (LA) above 5$15 \mu M$ [Fig. 3(A)]. A similar effect was also observed in the presence of $13(S)-\mathrm{H}(\mathrm{p}) \mathrm{ODE}$ [Fig. 3(C)]. The molecular basis for this inactivation has not been explored in detail, but substrate inhibition and/or suicidal inactivation by the reaction products may be discussed. In the 12/15-LOX monomers (PDB entry 2P0M) regardless of whether it is conformer $\mathrm{A}$ or $\mathrm{B}$, the side chains of the mutated residues would be mainly solvent exposed. Hence, it is unlikely that either of the point mutations at these positions significantly alters the packing of the active site upon substrate binding. In fact, when we created single mutants we hardly observed any effect of the mutations on the catalytic properties (data not shown). On the other hand, the correlation between the protein concentration and the amounts of substrate required for enzyme inactivation [Fig. 3(B), shown for W181E + H585E] suggested that direct substrate inhibition might be involved. As long as the two wild-type conformers A and $\mathrm{B}$ maintain their dimer interface, both hydrophobic $\alpha 2$ helixes are shielded from the solvent environment. However, once the interface is disturbed by the mutations, as it happens in the case of W181E + H585E and L183 + L192E, the $\alpha 2$ helices might come in contact with excessive amounts of fatty acid (LA) and/or allosteric ligand $(13(S)-\mathrm{H}(\mathrm{p}) \mathrm{ODE})$ to cause inhibition. This assumption is supported by our previous observations indicating a linkage of $15(\mathrm{~S})$-HpETE to $\alpha 2$ helix residues of rabbit $12 / 15-$ LOX $^{5}$ 

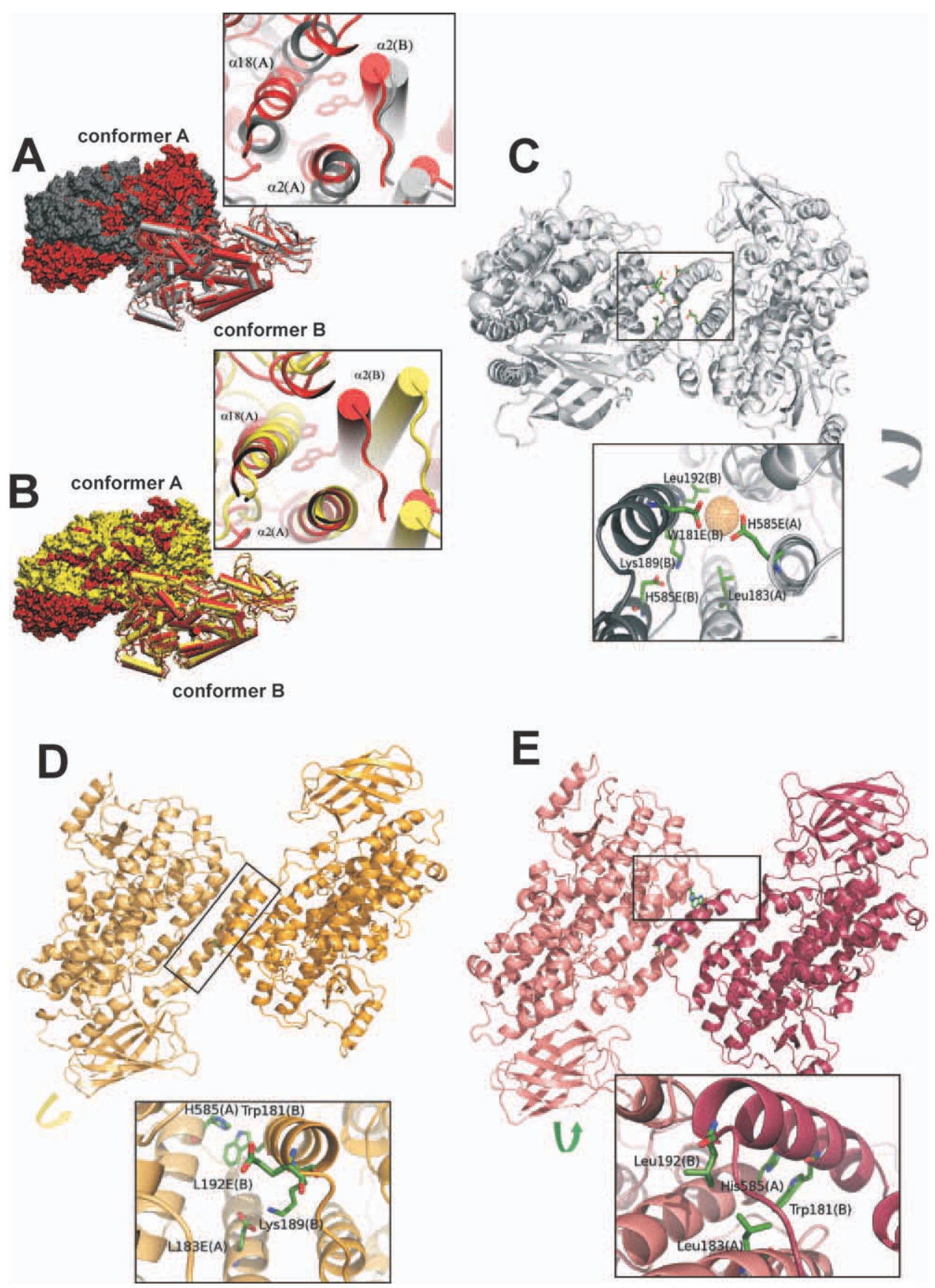

\section{Figure 4}

Structural comparison of the dimer interface. (A) Structural comparison of wild-type dimer (red) with W181E + H585E dimer mutant (gray) and (B) L183E + L192E dimer mutant (yellow) based on the MD simulations. To show the relative movement of conformer A (colored solid surfaces) with respect to conformer B, the alpha carbons of conformer B (colored cartoon) were used for the structural alignment of the dimers. The framed figure represents a close-up view of the dimers interface. W181 (conformer B) and H585 (conformer A) of the WT are represented in sticks. (C) Representative view of the W181E + H585E dimer from the 4-ns molecular dynamics simulations and zoom to the dimer interface. Conformers A and B are represented in gray ribbons, and residues Leu183(A), His585E(A), His585E(B), Trp181E(B), Leu192(B), and Lys189(B) are in green sticks. Spatial rearrangement of side chains of Glu181(B) and Glu585(A) is shown. (D) Representative view of the L183E + L192E dimer from the 4-ns molecular dynamics simulations and zoom to the dimer interface. Conformers A and B are represented in yellow ribbons, and residues Leu183E(A), Lys189(B), His585(A), Trp181(B), and Leu192E(B) are in green sticks. A salt-bridge formation between Lys189(B) and L183E(A) is observed, and His585(A) and Trp181(B) adopt a relative conformation in which their planes are perpendicular for part of the time. (E) Representative view of the wild-type dimer from the 4-ns molecular dynamics simulations and zoom to the dimer interface. The conformer A is depicted in light pink ribbons, conformer B in dark pink ribbons, and residues Leu183(A), His585(A), Trp181(B), and Leu192(B) in green sticks. In all these depictions, hydrogen atoms are not shown for clarity. The initial structure for the simulations of the dimer mutants is that of the WT enzyme dimer but with the mutated side chains. 
Molecular dynamics simulations of the enzyme-substrate complex for wild-type and double mutant dimers

To find out whether 12/15-LOX is able to form similar intermolecular associates in the presence of substrate and whether the proposed mutations disrupt the described interactions, we created in silico dimer models, in which conformer A is an "empty" molecule and conformer B contains AA at the active site. ${ }^{18}$ Similar models were created for the double mutants (W181E + H585E and L183E + L192E). Molecular dynamics simulations with periodic boundary conditions were carried out for the fully solvated wild-type enzyme dimer and for each of the mutants. Several free to move sodium cations were included to maintain the systems' neutrality. The analysis of the 4-ns trajectories for the W181E + H585E mutant revealed opening of the intermolecular interface [Fig. $4(\mathrm{~A}, \mathrm{C})$ ] along with rearrangement of E585 (conformer A) and E181 (conformer B) side chains to minimize electrostatic repulsions. Separation of the two mutated residues induces penetration of water molecules and eventually a sodium cation into the intermolecular crevice to stabilize this region [Fig. 4(C)]. The opening of the interface at that point is also affecting the interface at the hydrophobic part (leucine residues). In the 4-ns simulations, a rotation of conformer A relative to conformer B was observed, which clearly disrupts the original intermolecular interface and reduces its surface area from $1164 \pm 49 \AA^{2}$ (wild-type enzyme) to $693 \pm 10 \AA^{2}$ [Fig. 4(A)]. In the 4-ns trajectories for the L183E + L192E mutant, the reduction of the interface was also observed but somewhat less pronounced $\left(712 \pm 66 \AA^{2}\right)$. As it is shown in Figure 4(B), there is a significant structural rearrangement of the $\alpha 2$ helix of conformer $\mathrm{B}$, which contributes to the reduction of the intermolecular interface surface. Although the parallel $\pi-\pi$ interaction between H585 (monomer A) and W181 (monomer B) observed in the crystal structure is maintained part of the time, the two residues finally adopt other conformations in which their planes have rotated to be localized more perpendicular to each other [Fig. 4(D)]. Within the same simulation times, the wild-type dimer maintains the $\pi-\pi$ interaction between H585 (conformer A) and W181 (conformer B) along the whole trajectory, and the hydrophobic interaction on the dimer interface seems to be stable with the interface surface area of $1164 \pm 49 \AA^{2}$ [Fig. 4(E)]. Moreover, the $\alpha 2$ helix opens up as it did in some of our previous trajectories ${ }^{18}$ but maintaining the dimer interface.

In summary, our data indicate that allosteric effector 13(S)-HODE shifts the dynamic monomer-dimer equilibrium of 12/15-LOX favoring the formation of transient enzyme dimers. This dimeric complex seems to be also stable in the presence of the bound substrate (AA) as suggested by MD simulations. Interaction between the two flexible surface-exposed $\alpha 2$ helixes suggests that in the transient LOX dimer the two enzyme molecules might work in unison and, therefore, such mode of association might be related to the allosteric character of the enzyme. ${ }^{16,17}$ Introduction of negatively charged residues at the intermonomer interface (W181E + H585E and L183E + L192E mutants) disturbs hydrophobic interactions and leads to functional distortion of the mutant enzyme species. In addition, enzyme dimerization may protect the enzyme from kinetic substrate inhibition by shielding the hydrophobic $\alpha 2$ helixes.

\section{REFERENCES}

1. Funk CD. Prostaglandins and leukotrienes: advances in eicosanoid biology. Science 2001;294:1871-1875.

2. de Luca C, Olefsky JM. Inflammation and insulin resistance. FEBS Lett 2008;582:97-105.

3. Sears DD, Miles PD, Chapman J, Ofrecio JM, Almazan F, Thapar D, Miller YI. 12/15-lipoxygenase is required for the early onset of high fat diet-induced adipose tissue inflammation and insulin resistance in mice. PLoS One 2009;4:e7250.

4. Klein RF, Allard J, Avnur Z, Nikolcheva T, Rotstein D, Carlos AS, Shea M, Waters RV, Belknap JK, Peltz G, Orwoll ES. Regulation of bone mass in mice by the lipoxygenase gene Alox15. Science 2004;303:229-232.

5. Wiesner R, Suzuki H, Walther M, Yamamoto S, Kuhn H. Suicidal inactivation of the rabbit 15-lipoxygenase by $15 \mathrm{~S}$-HpETE is paralleled by covalent modification of active site peptides. Free Radic Biol Med 2003;34:304-315.

6. Hammel M, Walther M, Prassl R, Kuhn H. Structural flexibility of the N-terminal beta-barrel domain of 15-lipoxygenase-1 probed by small angle X-ray scattering. Functional consequences for activity regulation and membrane binding. J Mol Biol 2004;34:917-929.

7. Ludwig P, Holzhütter H-G, Colosimo A, Silvestrini MC, Schewe T, Rapoport SM. A kinetic model for lipoxygenases based on experimental data with the lipoxygenase of reticulocytes. Eur J Biochem 1987;168:325-337.

8. Shang W, Ivanov I, Svergun DI, Borbulevych OY, Aleem AM, Stehling S, Jankun J, Kuhn H, Skrzypczak-Jankun E. Probing dimerization and structural flexibility of mammalian lipoxygenases by small angle X-ray scattering. J Mol Biol 2011;409:654-668.

9. Choi J, Chon JK, Kim S, Shin W. Conformational flexibility in mammalian 15S-lipoxygenase: reinterpretation of the crystallographic data. Proteins 2008;70:1023-1032.

10. Schulz EC, Tietzel M, Tovy A, Ankri S, Ficner R. Structure analysis of Entamoeba histolytica enolase. Acta Cryst D 2011;67:619-627.

11. Nooren IMA, Thornton JM. Structural characterisation and functional significance of transient protein-protein interactions. J Mol Biol 2003;325:991-1018.

12. Chung I, Akita R, Vandlen R, Toomre D, Schlessinger J, Mellman I. Spatial control of EGF receptor activation by reversible dimerization on living cells. Nature 2010;464:783-787.

13. Tateyama M, Abe H, Nakata H, Saito O, Kubo Y. Ligand-induced rearrangement of the dimeric metabotropic glutamate receptor 1alpha. Nat Struct Mol Biol 2004;11:637-642.

14. Yuan C, Rieke CJ, Rimon G, Wingerd BA, Smith WL. Partnering between monomers of cyclooxygenase-2 homodimers. Proc Natl Acad Sci USA 2006;103:6142-6147.

15. Yuan C, Sidhu RS, Kuklev DV, Kado Y, Wada M, Song I, Smith WL. Cyclooxygenase allosterism, fatty acid-mediated cross-talk between monomers of cyclooxygenase homodimers. J Biol Chem 2009;284:10046-10055.

16. Wecksler AT, Kenyon V, Deschamps JD, Holman TR. Substrate specificity changes for human reticulocyte and epithelial 15-lipoxy- 
genases reveal allosteric product regulation. Biochemistry 2008;47:7364-7375.

17. Wecksler AT, Jacquot C, van der Donk WA, Holman TR. Mechanistic investigations of human reticulocyte 15- and platelet 12-lipoxygenases with arachidonic acid. Biochemistry 2009;48:6259-6267.

18. Toledo L, Masgrau L, Maréchal JD, Lluch JM, González-Lafont À. Insights into the mechanism of binding of arachidonic acid to mammalian 15-lipoxygenases. J Phys Chem B 2010;114:7037-7046.

19. Roessle MW, Klaering R, Ristau U, Robrahn B, Jahn D, Gehrmann T, Konarev P, Round A, Fiedler S, Hermesa C, Svergun DI. Upgrade of the small-angle X-ray scattering beamline X33 at the European Molecular Biology Laboratory, Hamburg. J Appl Cryst 2007;40:190-194.

20. Round AR, Franke D, Moritz S, Huchler R, Fritsche M, Malthan D, Klaering R, Svergun DI, Roessle M. Automated sample-changing robot for solution scattering experiments at the EMBL Hamburg SAXS station X33. J Appl Cryst 2008;41:913-917.

21. Konarev PV, Volkov VV, Sokolova AV, Koch MHJ, Svergun DI. PRIMUS: a Windows PC-based system for small-angle scattering data analysis. J Appl Crystallogr 2003;36:1277-1282.

22. Petoukhov MV, Svergun DI. Global rigid body modeling of macromolecular complexes against small-angle scattering data. Biophys J 2005;89:1237-1250.

23. Svergun DI, Barberato C, Koch MHJ. CRYSOL-a program to evalate X-ray solution scattering of biological macromolecules from atomic coordinates. J Appl Crystallogr 1995;28:768-773.

24. Brooks BR, Brooks CL, Mackerell AD, Nilsson L, Petrella RJ, Roux B, Won Y, Archontis G, Bartels C, Boresch S, Caflisch A, Caves L, Cui Q, Dinner AR, Feig M, Fischer S, Gao J, Hodoscek M, Im W, Kuczera K, Lazaridis T, Ma J, Ovchinnikov V, Paci E, Pastor RW, Post CB, Pu JZ, Schaefer M, Tidor B, Venable RM, Woodcock HL, Wu X, Yang W, York DM, Karplus M. CHARMM: the biomolecular simulation program. J Comput Chem 2009;30:1545-1614.

25. Pettersen EF, Goddard TD, Huang CC, Couch GS, Greenblatt DM, Meng EC, Ferrin TE. UCSF Chimera-a visualization system for exploratory research and analysis. J Comput Chem 2004;25:1605-1612.

26. Hoover WG. Canonical dynamics: equilibrium phase-space distributions. Phys Rev A 1985;31:1695-1697.
27. Ryckaert JP, Ciccotti G, Berendsen HJC. Numerical integration of the cartesian equations of motion of a system with constraints: molecular dynamics of n-alkanes. J Comput Phys 1977;23:327-341.

28. MacKerell AD, Bashford D, Bellott M, Dunbrack RL, Evanseck JD, Field MJ, Fischer S, Gao J, Guo H, Ha S, Joseph-McCarthy D, Kuchnir L, Kuczera K, Lau FTK, Mattos C, Michnick S, Ngo T, Nguyen DT, Prodhom B, Reiher WE, Roux B, Schlenkrich M, Smith JC, Stote R, Straub J, Watanabe M, Wiorkiewicz-Kuczera J, Yin D, Karplus M. All-atom empirical potential for molecular modeling and dynamics studies of proteins. J Phys Chem B 1998;102:3586-3616.

29. MacKerell AD, Feig M, Brooks CL. Extending the treatment of backbone energetics in protein force fields: limitations of gas-phase quantum mechanics in reproducing protein conformational distributions in molecular dynamics simulations. J Comput Chem 2004;25:1400-1415.

30. Saam J, Ivanov I, Walther M, Holzhutter H-G, Kuhn H. Molecular dioxygen enters the active site of 12/15-lipoxygenase via dynamic oxygen access channels. Proc Natl Acad Sci USA 2007;104:1331913324.

31. Feller SE, MacKerell AD. An improved empirical potential energy function for molecular simulations of phospholipids. J Phys Chem B 2000;104:7510-7515.

32. Ivanov I, Di Venere A, Horn T, Scherer P, Nicolai E, Steling S, Richter C, Skrzypczak-Jankun E, Mei G, Maccarrone M, Kühn H. Tight association of N-terminal and catalytic subunits of rabbit 12/ 15-lipoxygenase is important for protein stability and catalytic activity. Biochim Biophys Acta 2011;1811:1001-1010.

33. Nikolaev Y, Pervushin K. Rethinking leucine zipper-a ubiquitous signal transduction motif. Nat Preced 2009. Available at: hdl:10101/ npre.2009.3271.1.

34. Walther M, Anton M, Wiedmann M, Fletterick R, Kuhn H. The Nterminal domain of the reticulocyte-type 15-lipoxygenase is not essential for enzymatic activity but contains determinants for membrane binding. J Biol Chem 2002;277:27360-27366.

35. Humphrey W, Dalke A, Schulten K. VMD: visual molecular dynamics. J Mol Graph 1996;14:33-38. 